\title{
Validating weather and climate models at small Rossby numbers: including a boundary layer
}

\author{
Robert J. Beare ${ }^{1 *}$ and Michael J. P. Cullen ${ }^{2}$ \\ $1 *$ Department of Mathematics, University of Exeter, UK. \\ ${ }^{2}$ Met Office, Exeter, UK
}

${ }^{*}$ Correspondence to: Department of Mathematics, University of Exeter, United Kingdom. EX4 4QF. Email: r.j.beare@ex.ac.uk

Ideally, the validation of weather and climate models requires that the predictions remain close to an exact solution of the governing equations. The complexity of weather and climate models means that it is not possible to compute exact solutions except in trivial cases. However, in the limit of small Rossby number, the exact solution of the Euler equations can be shown to be close to that of a semi-geostrophic model, which can be computed. Previous studies have used the small Rossby-number limit to validate numerical methods for a baroclinic wave without sub-grid physics. However, the method of coupling to the sub-grid physics plays an important role in the performance of weather and climate models. The aim of this paper is thus to extend the previous studies to include a boundary-layer parametrization. We use a balanced model that includes a known boundary-layer parametrization, the semi-geotriptic model. We then demonstrate that the semi-geotriptic model is the appropriate small Rossby-number limit of the solution of the Euler equations with the same boundary layer representation. The semi-geotriptic model is then used to expose weaknesses in the numerical methods for coupling the boundary layer to the rest of the model.

Key Words: Validation; Ekman balance; Boundary layer; Advection; Semi-geotriptic model; Timestepping

Received; Revised ; Accepted

1. Introduction

Exact solutions provide the ideal reference for validating weather and climate models. It is not currently possible to compute exact solutions except in trivial cases because the computations required would be impracticable. Production numerical models use an implicitly averaged form of the equations together with a range of sub-grid parametrizations that account for processes not resolved by the model grid. These include: the boundary layer, moist 
convection, gravity waves and the land surface. Moreover, subgrid processes couple to the resolved dynamics in a complicated way that is not presently fully understood (Cullen and Salmond 2003). The error introduced by this averaging cannot be usefully estimated theoretically.

Under certain limiting conditions, the exact solution of the Euler equations can be shown to be the same as that of a simpler model, which can be computed. These solutions are thus valuable for validating prediction models. In the limit of small Rossby number (Ro), and in the absence of sub-grid processes, the exact solution of the Euler equations have been shown to converge to that of a semi-geostrophic (SG) model (Cullen 2007). For a twodimensional baroclinic wave case and in the absence of boundarylayer diffusion, Cullen (2008) showed that computed solutions of the SG and Euler solutions converged with decreasing Ro at a second-order rate. Recently, Visram et al. (2014) validated a semi-Lagrangian advection scheme for a similar case by testing for second-order convergence to the SG solution. Here the SG solution is the exact asymptotic limit of the Euler solution as Ro tends to zero.

The need to enforce the no-slip condition at the lower boundary requires a fundamental change to this procedure, even if the primary interest is large-scale flows. For instance, the boundary layer can play an order-one role in the development of baroclinic waves (Beare 2007). In previous work, we have extended the SG model to include a no-slip boundary condition by coupling it to a standard one-dimensional boundary-layer formulation, which should be sufficient on scales where the SG model is appropriate (Beare and Cullen 2013). This is called the semi-geotriptic (SGT) model. We previously demonstrated the use of the SGT model in understanding: sea breezes, tropical convergence zones and midlatitude frontal jets (Cullen 1989; Beare and Cullen 2010, 2012, 2013). We also showed that solutions of the shallow water system converged to SGT solutions at a first order rate in Rossby number (Beare and Cullen 2012). However, the equivalent calculation remains to be done for three-dimensional models.

Ideally, we need to show that the SGT model is the small-Ro limit of solutions of the Navier-Stokes equations with the usual no-slip boundary condition. Whilst proving this mathematically is currently too ambitious, in this paper we intend to make a useful contribution, showing large-scale numerical models including a boundary-layer parametrization respect this limit. Classical fluid dynamics treats the Euler equations in the interior of the fluid with a one-dimensional boundary layer model matched to it close to the boundary as an asymptotic limit of the Navier-Stokes equations (e.g. Batchelor 2000). In the atmosphere, this theory has to be extended to include boundary layers at higher Reynolds numbers, using a boundary-layer diffusion with magnitude dependent on the shear and stratification (Garratt 1992). Whilst the magnitude of the effects of stratification remain uncertain (e.g Beare et al. 2006), the near-neutral, small-stratification limit is relatively well understood. We therefore assume that the validity of a standard boundary layer formulation has been established for a near-neutral scenario. We then show formally that if this formulation is used in the SGT model, the validity of SG as the small-Ro limit of the Euler equations extends to the validity of SGT as the small-Ro limit of the Navier-Stokes equations. This analysis is performed using the hydrostatic primitive equations (HPEs), as these will be an accurate approximation to the Euler equations whenever SGT is applicable. The analyses of Bannon (1998), Snyder (1998) and Tory and Reeder (2005) indicated the importance of Ro for scaling advection when a boundary layer is included. We will perform a scale analysis that shares some aspects with these studies, but also identifies the temporal and Froude number regimes under which the Ro controls the convergence of the HPE model solutions to those of SGT.

Numerical models, which are used on large enough horizontal scales for a one-dimensional boundary layer formulation to be appropriate, can also use the hydrostatic approximation. Thus we examine the limiting behaviour of a model using the HPEs with a boundary layer scheme as Ro decreases. If the HPEs with a boundary layer correctly represented the averaged behaviour of the Navier-Stokes equations, they would have solutions that are smooth on the averaging scale and thus computable. However we do not investigate this issue, but regard the numerical computations with a sufficiently small timestep as our best estimate of the averaged solutions. We then seek to validate these solutions by investigating their behaviour as Ro decreases. If they show the expected convergence to SGT, and the boundary layer model can be assumed to be accurate, then the numerical model 
will be exhibiting the correct large-scale behaviour. We will use a baroclinic-wave test case as it will allow direct comparison with the previous SG studies. As with the SG problem, the rate of convergence wil also be valuable for validation purposes.

We then aim to validate the HPE model at larger timesteps using our understanding of the rates of convergence to the SGT model. Increasing the timestep in the HPE model presents more of a challenge for the numerical methods as the time variation of the boundary-layer diffusion coefficient becomes important. Such variation is a typical situation in weather and climate models, where the timestep can only be reduced so much in the interest of producing timely predictions (Diamantakis et al. 2006). In situations where the convergence is disrupted, our method indicates areas for model improvement.

\section{Scale and convergence analysis}

Before we proceed to the analysis of baroclinic wave simulations, we need to establish when the Rossby number controls the convergence of the HPE solutions to SGT solutions. We do this by presenting a scale analysis of the HPEs, including a boundarylayer parametrization. We then define the approximations made to the HPEs in deriving the SGT model. In the following, the key scale assumptions are displayed in boxes.

\subsection{Key dimensionless parameters}

The Boussinesq HPEs on an $f$-plane, including boundary-layer parametrization terms are:

$$
\begin{gathered}
\frac{D \mathbf{u}}{D t}+f \mathbf{k} \times \mathbf{u}+\nabla \phi=\frac{\partial}{\partial z}\left(K_{m} \frac{\partial \mathbf{u}}{\partial z}\right), \\
\frac{\partial \phi}{\partial z}=g \frac{\theta-\theta_{0}}{\theta_{0}}, \\
\frac{D \theta}{D t}=\frac{\partial}{\partial z}\left(K_{h} \frac{\partial \theta}{\partial z}\right), \\
\nabla \cdot \mathbf{u}+\frac{\partial w}{\partial z}=0 \quad \text { and } \\
\frac{D}{D t}=\frac{\partial}{\partial t}+\mathbf{u} \cdot \nabla+w \frac{\partial}{\partial z} .
\end{gathered}
$$

The symbols used above are defined in Table 1. Vector values are in bold and scalars are in italics throughout.

\begin{tabular}{cc}
\hline Symbol & Meaning \\
\hline$t$ & Time \\
$D$ & Material derivative \\
$\mathbf{u}$ & Horizontal wind vector \\
$f$ & Coriolis parameter (constant) \\
$\mathbf{k}$ & Unit vertical vector \\
$\times$ & Cross product \\
$\nabla$ & Horizontal vector gradient \\
$\phi$ & Geopotential \\
$z$ & Height coordinate, pseudo-height \\
& (Hoskins and Bretherton 1972) \\
$g$ & Gravitational acceleration \\
$\theta_{0}$ & Potential temperature \\
$K_{m}$ & Boundary-layer vertical momentum diffusivity \\
$K_{h}$ & Boundary-layer vertical heat diffusivity \\
$w$ & Vertical wind \\
\hline
\end{tabular}

Table 1. Symbols used in Eqs. (1) - (5).

\subsubsection{Momentum equation above boundary layer}

We non-dimensionalise Eqs. (1) - (5) using the scales: horizontal wind $(U)$, vertical wind $(W)$, horizontal length scale $(L)$, depth of the troposphere $(H)$, Brunt-Väisällä frequency $(N)$ and geopotential $\left(N^{2} H^{2}\right)$. We assume that horizontal and vertical gradients of geopotential are related by the factor $H / L$, which excludes scalings based on a uniform reference profile. The latter is not appropriate for considering the boundary layer. All dimensionless variables are assumed to be order-one in magnitude (notated as $\mathcal{O}(1)$, where $\mathcal{O}$ denotes the order). Changes in magnitude are then reflected in the scaling parameters (such as Ro) that form the coefficients of the dimensionless quantities. We now seek to make consistent choices of scaling parameters.

We presume the existence of solutions that converge to geostrophic balance above the boundary layer in the limit of small Ro. We thus select a timescale for advection $(T)$ that satisfies

$$
T \sim \frac{L}{U} \sim \frac{H}{W}
$$

so that each term of the material derivative (Eq. 5) scales in the same way. The slow timescale enforced by Eq. (6) means that fast waves, such as gravity waves, are excluded. In contrast, higher-order balanced models do not enforce Eq. (6) (Cullen 2006, section 2.4.3). The dimensionless form of Eq. (1) is

$$
\overbrace{\operatorname{Ro} \frac{D \widehat{\mathbf{u}}}{D \widehat{t}}}^{\text {Material Derivative }}+\overbrace{\mathbf{k} \times \widehat{\mathbf{u}}}^{\text {Coriolis }}+\overbrace{\frac{\mathrm{Ro}}{\mathrm{Fr}^{2}} \widehat{\nabla} \widehat{\phi}}^{\text {Pressure gradient }}=0
$$


where ${ }^{-}$indicates dimensionless values and the names of the terms are annotated above for future reference. The definitions of Ro and the Froude number (Fr) are

$$
\begin{aligned}
& \text { Ro }=\frac{U}{f L} \text { and } \\
& \operatorname{Fr}=\frac{U}{N H} .
\end{aligned}
$$

For small Ro, the Coriolis and pressure gradient terms in Eq. (7) should balance, giving

$$
\mathrm{Ro}=\mathrm{Fr}^{2}
$$

Equation (7) simplifies to

$$
\operatorname{Ro} \frac{D \widehat{\mathbf{u}}}{D \widehat{t}}+\mathbf{k} \times \widehat{\mathbf{u}}+\widehat{\nabla} \widehat{\phi}=0
$$

In the limit of Ro $\rightarrow 0$, Eq. (11) satisfies geostrophic balance

$$
\mathbf{k} \times \widehat{\mathbf{u}}_{\mathbf{g}}+\widehat{\nabla} \widehat{\phi}=0
$$

where $\widehat{\mathbf{u}}_{\mathbf{g}}$ is the geostrophic wind.

\subsubsection{Boundary-layer momentum equation}

We showed previously that Ro controls the deviation from geostrophic balance above the boundary layer. We now consider the parameters determining Ekman balance within the boundary layer. The relevant vertical scale is the boundary-layer depth $(h)$, defined as depth where the parametrized fluxes fall to zero. In general, $h$ varies in the horizontal so we use $\bar{h}$, where the overbar indicates the horizontal mean, as the representative scale. We also define $K$ as the scale for the boundary-layer diffusion. The boundary-layer drag scales as

$$
\frac{\partial}{\partial z}\left(K_{m} \frac{\partial \mathbf{u}}{\partial z}\right) \sim \frac{K U}{\bar{h}^{2}}
$$

The timescale is assumed to be given by the advective timescale in the boundary layer as well as above it, following Eq. (6). Fast waves are therefore excluded in the boundary layer as they are above it. Each term of the material derivative (Eq. 5) within the boundary layer scales in the same way as in Eq. (6). The dimensionless form of Eq. (1) within the boundary layer is now

$$
\overbrace{\operatorname{Ro} \frac{D \widehat{\mathbf{u}}}{D \widehat{t}}}^{\text {Material Derivative }}+\overbrace{\mathbf{k} \times \widehat{\mathbf{u}}}^{\text {Coriolis }}+\overbrace{\widehat{\nabla} \widehat{\phi}}^{\text {Pressure gradient }}=\overbrace{\mathrm{Ek} \hat{B} \widehat{\mathbf{u}}}^{\text {Drag }}
$$

where the Ekman (Ek) number is defined as

$$
\mathrm{Ek}=\frac{K}{f \bar{h}^{2}}
$$

The dimensionless boundary-layer diffusion operator $(\mathcal{B})$ is given by

$$
\mathcal{B}=\bar{h}^{2} \frac{\partial}{\partial z} \widehat{K}_{m} \frac{\partial}{\partial z}
$$

We assume shallow boundary layers $(\bar{h} \ll H)$ and hydrostatic balance. The change of the pressure gradient across the boundary layer is therefore negligible compared to the change over the depth of the troposphere. The no-slip boundary condition means that the Coriolis and drag terms do vary in the vertical and have to balance each other in the limit of small Ro (Eq. 14), giving

$$
\mathrm{Ek}=\mathcal{O}(1)
$$

Equation (14) simplifies to

$$
\operatorname{Ro} \frac{D \widehat{\mathbf{u}}}{D \widehat{t}}+\mathbf{k} \times \widehat{\mathbf{u}}+\widehat{\nabla} \widehat{\phi}=\mathcal{B} \widehat{\mathbf{u}}
$$

Due to the timescale assumption (Eq. 6), the form of the material derivative is the same in Eqs. (11) and (18). In the limit of Ro $\rightarrow 0$, Eq. (18) satisfies Ekman balance, given by

$$
\mathbf{k} \times \widehat{\mathbf{u}}_{\mathbf{e}}+\widehat{\nabla} \widehat{\phi}=\quad \mathcal{B} \widehat{\mathbf{u}}_{\mathbf{e}}
$$

where $\widehat{\mathbf{u}}_{\mathbf{e}}$ is the Ekman momentum. It is important to note that $\widehat{\mathbf{u}}_{\mathbf{e}} \rightarrow \widehat{\mathbf{u}}_{\mathbf{g}}$ above the boundary layer, so the Ekman momentum includes the geostrophic momentum.

We assume that $K_{m}$ is driven by vertical wind shear and so a function of the wind at the top of the boundary layer. Since we have constrained Ek (Eqs. 15 and 17), it follows that $\bar{h}$ is a function of Ro

$$
\bar{h}=\bar{h}(\mathrm{Ro})
$$


Height

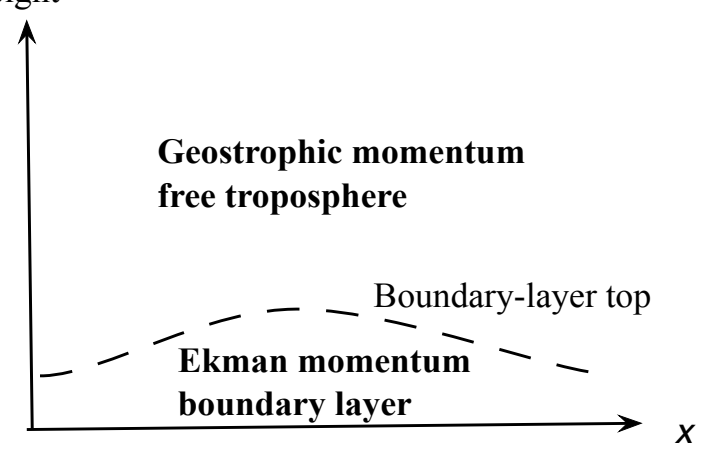

Figure 1. A schematic of the SGT model. It is close to Ekman balance within the boundary layer, and semi-geostrophic (geostrophic momentum) above.

Related scale analyses have been performed by Snyder (1998) and Tory and Reeder (2005). In contrast, here we have been explicit in our timescale assumptions and in constraining Fr and Ek so that Ro is a single controlling parameter. We have also included the dependence of the boundary-layer depth on Ro.

\subsection{SGT model}

Ostdiek and Blumen (1997) analysed observations of frontal boundary layers. Although the SG model was valid above the boundary layer, its assumptions broke down within it. The SGT model combines the SG model with a boundary layer, as illustrated in Fig. 1. The SGT model maintains an Ekmanbalanced boundary layer in the presence of a time-varying SG solution above. The dimensionless framework will help us to understand the approximations made to the HPE model in deriving the SGT model.

Just as the SG model uses the geostrophic momentum as a prognostic variable, the SGT model uses the Ekman momentum. Using the Ekman momentum, the SGT model diagnoses a trajectory with components $\widehat{\mathbf{u}}_{\mathbf{s}}$ in the horizontal and $\widehat{w}_{s}$ in the vertical. In deriving the SGT model, two key approximations are applied to Eq. (18). First, the material derivative is approximated as

$$
\operatorname{Ro} \frac{D \widehat{\mathbf{u}}}{D \widehat{t}}=\operatorname{Ro} \frac{D_{s} \widehat{\mathbf{u}}_{\mathbf{e}}}{D \widehat{t}}+\mathcal{O}\left(\operatorname{Ro}^{2}\right)
$$

where

$$
\frac{D_{s}}{D \widehat{t}}=\frac{\partial}{\partial \widehat{t}}+\widehat{\mathbf{u}}_{\mathbf{s}} \cdot \nabla+\widehat{w}_{s} \frac{\partial}{\partial \widehat{z}}
$$

The $\mathcal{O}\left(\mathrm{Ro}^{2}\right)$ in Eq. (21) implies that there will be a secondorder deviation of the HPE model from the SGT model. The second approximation is applied to the drag term in Eq. (11). Given that Ekman momentum is now a prognostic variable, Beare and Cullen (2012) and Beare and Cullen (2013) showed that a second-order approximation of the drag term leads to a physically unrealistic non-decaying energy equation. However, a realistic energy evolution was recovered by using the first-order approximation

$$
\mathcal{B} \widehat{\mathbf{u}}=\mathcal{B} \widehat{\mathbf{u}}_{\mathbf{e}}+\overbrace{\mathcal{B}\left(\widehat{\mathbf{u}}_{\mathbf{e}}-\widehat{\mathbf{u}}_{\mathbf{s}}\right)}^{\text {Relaxation }}+\mathcal{O}(\mathrm{Ro})=\mathcal{B}\left(2 \widehat{\mathbf{u}}_{\mathbf{e}}-\widehat{\mathbf{u}}_{\mathbf{s}}\right)+\mathcal{O}(\mathrm{Ro}) .
$$

The annotated 'relaxation' term is first-order accurate in Ro; it relaxes the Ekman momentum to the trajectory in time. Given zero vertical velocity boundary conditions at the top and bottom of the domain, the relaxation to Ekman balance also ensured a no-slip surface boundary condition. Combining Eqs. (21) and (23) gives the SGT momentum balance

$$
\overbrace{\operatorname{Ro} \frac{D_{s} \widehat{\mathbf{u}}_{\mathbf{e}}}{D \widehat{t}}}^{\text {2nd order accurate }}+\mathbf{k} \times \widehat{\mathbf{u}}_{\mathbf{s}}+\widehat{\nabla} \widehat{\phi}=\overbrace{\mathcal{B}\left(2 \widehat{\mathbf{u}}_{\mathbf{e}}-\widehat{\mathbf{u}}_{\mathbf{s}}\right)}^{1 \text { st order accurate }}
$$

Equation (24) is a mathematical realisation of the schematic in Fig. 1. In the boundary layer, a solution close to Ekman balance is maintained; above the boundary layer, the solution matches the geostrophic momentum approximation of the SG model given by

$$
\operatorname{Ro} \frac{D_{s} \widehat{\mathbf{u}}_{\mathbf{g}}}{D \widehat{t}}+\mathbf{k} \times \widehat{\mathbf{u}}_{\mathbf{s}}+\widehat{\nabla} \widehat{\phi}=0
$$

\subsection{Convergence of HPE to SGT}

The previous analysis is based on scale assumptions that need to be shown to define a solution which is an asymptotic limit of the HPEs with a boundary layer, and thus of the Euler equations with the same boundary layer formulation. A mathematical proof would be ideal, but does not currently exist. Instead we used small-timestep HPE simulations to demonstrate convergence to an SGT solution as Ro decreases. The Ekman momentum and SGT trajectory was diagnosed from these simulations (see appendix). The difference between HPE and SGT solutions was measured by diagnosing

$$
\left\|\widehat{\mathbf{u}}_{\mathbf{s}}-\widehat{\mathbf{u}}\right\|
$$


where || || denotes a root-mean-square (rms) domain average, and where $\widehat{\mathbf{u}}$ is from the small timestep HPE simulations. Assuming our scale assumptions are correct, the term with the least accuracy will dominate the reduction of the difference between the HPE and SGT models as Ro decreases. Within the boundary layer, the firstorder assumption should dominate (Eq. 23). In contrast, above the boundary layer, the convergence is only limited by the secondorder assumption (Eq. 21). In order to isolate these dependencies, we calculated Eq. (26) for separate sub-domains within and above the boundary layer. When averaging over the entire domain, the boundary-layer depth (Eq. 20) determines the relative volume occupied by the boundary layer in the domain integral. We thus postulate that, for the domain integral,

$$
\left\|\widehat{\mathbf{u}}_{\mathbf{s}}-\widehat{\mathbf{u}}\right\| \propto \bar{h} R o
$$

The boundary-layer depth is often controlled by the vertical shear for a near-neutral scenario. The vertical shear is a function of $U$ and thus Ro. It is thus reasonable to postulate a power law for the mean boundary-layer depth

$$
\bar{h} \propto \mathrm{Ro}^{m}
$$

where $m$ is a positive power. The existence of the power law was tested in our simulations.

\section{Baroclinic wave case}

We now describe the baroclinic wave test case. First, we define the $2 \mathrm{D}$ version of the HPE model. We then define the rescaling of fields to achieve different values of Ro. The method of calculating the difference between the small timestep HPE solutions and those from the SGT model is then given. Finally, we repeat the calculations using the HPE model at larger timesteps.

\subsection{HPE model}

We used numerical simulations of the HPEs (Eqs. 1-5) on a 2D vertical slice $(x, z)$. The components of the horizontal wind were

$$
\begin{aligned}
& u=u_{g}(z)+u_{a}(x, z, t), \\
& v=v_{g}(x, z, t)+v_{a}(x, z, t),
\end{aligned}
$$

where $\left(u_{g}, v_{g}\right)$ and $\left(u_{a}, v_{a}\right)$ are the geostrophic and ageostrophic horizontal wind vectors respectively and $u_{g}$ is a function of height only. The model was configured for a mid-latitude baroclinic wave coupled to a boundary-layer parametrization. The boundary-layer scheme is defined in the appendix (Eqs. 45-47). Otherwise, the configuration was similar to that of Keyser and Anthes (1982) and Tory and Reeder (2005). The geostrophic basic state was defined relative to the mid-level winds

$$
u_{g}=\frac{2 u_{0}}{H}(z-H / 2)
$$

so that the large-scale wave remained stationary. The actual winds in $x$ were recovered by the transform $u \rightarrow u+u_{0}$. The horizontal velocity scale used in the scale analysis (section 2) was chosen as $U=u_{0}$. The value of $U$ was important in controlling the magnitude of the advection in the $x$-direction. The equations solved were

$$
\frac{D u}{D t}-f\left(v-v_{g}\right)=\frac{\partial}{\partial z}\left(K_{m} \frac{\partial u}{\partial z}\right)
$$

$$
f v_{g}=\frac{\partial \phi}{\partial x}
$$

$$
\frac{D v}{D t}+f\left(u-u_{g}\right)=\frac{\partial}{\partial z}\left(K_{m} \frac{\partial v}{\partial z}\right)
$$

$$
f \frac{\partial u_{g}}{\partial z}=-\frac{g}{\theta_{0}} \frac{\partial \Theta}{\partial y}
$$

$$
\frac{\partial \phi}{\partial z}=g \frac{\theta-\theta_{0}}{\theta_{0}}
$$

$$
\begin{gathered}
\frac{D \theta}{D t}=\frac{\partial}{\partial z}\left(K_{h} \frac{\partial \theta}{\partial z}\right)-v \frac{\partial \Theta}{\partial y} \\
\frac{\partial u}{\partial x}+\frac{\partial w}{\partial z}=0 \\
\frac{D}{D t}=\frac{\partial}{\partial t}+u \frac{\partial}{\partial x}+w \frac{\partial}{\partial z}
\end{gathered}
$$

where $\frac{\partial \Theta}{\partial y}$ is a constant basic state potential temperature gradient in the $y$-direction. The advection across the basic-state potential temperature gradient is the last term on the right hand side of Eq. (37).

The lateral boundary conditions were periodic. At the bottom boundary, we defined no-slip conditions for momentum and followed Keyser and Anthes (1982) by using simplified insulating 
conditions for the potential temperature

$$
u_{a}=v=\frac{\partial \theta}{\partial z}=0 \quad \text { at } \quad z=0
$$

The vertical velocity boundary conditions were

$$
w=0 \quad \text { at } \quad z=0 \text { and } H
$$

\subsection{Numerical solution of HPE equations}

The dynamics was solved using the three-time-level method of Williams (1967), except that a more numerically stable semiLagrangian advection scheme was used. The Lorenz grid was used in the vertical with 51 levels and a quadratic variation of grid length to give additional resolution in the boundary layer. The bottom level (first calculation point) was $5 \mathrm{~m}$, and there were 11 levels within the first $500 \mathrm{~m}$. In the horizontal, 162 equally spaced points were used. The following scales were used: $H=9 \mathrm{~km}, L=$ $2000 \mathrm{~km}$ and $N=0.011 \times 10^{-2} \mathrm{~s}^{-1}$.

For each simulation, the fields $v, v_{g}$ and $\theta$ were initialised with the fastest-growing Eady wave (Williams 1967). The ageostrophic circulation $\left(u_{a}, w\right)$ was initialised using the Sawyer-Eliassen equation (Sawyer 1956; Eliassen 1962), given by Eq. (48) in the appendix. Short, 12 hour, simulations were performed giving the 'smooth' early growth phase of the baroclinic development; others have considered the discontinuous frontal collapse for longer integrations using just the dynamics (Visram et al. 2014). However, Snyder (1998) found that, when the wind-turning from a boundary layer is included, the flow can deviate substantially from Ekman balance for a strong front. Thus short integrations were the most appropriate for calculating asymptotic limits.

\subsection{Rescaling fields}

In order to sample a range of Ro whilst satisfying the scaling assumptions of Section 2, we employed the following rescaling of variables. We fixed the initial potential temperature field and the horizontal length scale $L$. In order to also maintain initial thermal wind balance, we ensured the product $f u_{g}$ was constant in the initial fields. In the SG case, the geostrophic wind in the $y$-direction was fixed (Cullen 2008). However, including a boundary layer generates a component of the Ekman momentum in the $x$-direction, the so-called wind turning. There is therefore no preferred direction, and the wind in all horizontal directions must be rescaled. We rescale the winds and Coriolis parameter in the following way, using a factor $\alpha$

$$
u_{g} \rightarrow \alpha u_{g}, \quad\left(v, v_{g}\right) \rightarrow \alpha\left(v, v_{g}\right), \quad f \rightarrow f / \alpha .
$$

The Rossby and Froude numbers scale as

$$
\mathrm{Ro} \sim u_{g} / f \sim \alpha^{2}, \quad \text { Fr } \sim u_{g} \sim \alpha, \quad \text { Ro }=\mathrm{Fr}^{2},
$$

where Fr only depends on wind speed as the initial value of $N$ is fixed. Equation (43) thus ensured that, whilst varying Ro, the simulations remained in the regime defined by Eq. (10).

The value of $\alpha^{2}$ was varied between 0.5 and 2 in increments of 0.125 . For $\alpha=1$, the values of $u_{0}=14.7 \mathrm{~ms}^{-1}, f=10^{-4} \mathrm{~s}^{-1}$ and amplitude of $v_{g}$ of $3.87 \mathrm{~ms}^{-1}$ were used. A range of Ro from 0.037 to 0.15 resulted. The range was smaller than is possible for cases without sub-grid physics (Visram et al. 2014) due to the variation of boundary-layer depth with Ro. Nevertheless, a factor of 4 in Ro was covered.

\subsection{Validating the large-scale behaviour of the HPE model}

The difference between the HPE and SGT models was calculated using the following steps:

\section{HPE model run at small timestep (control HPE} simulation). Simulations were repeated over the range of Ro.

2. SGT solution diagnosed from each control HPE simulation. See appendix (Eqs. 51-55) for details.

3. Difference between control HPE simulations and SGT solutions calculated, and quantified using Eq. (26).

\subsection{Validating HPE simulation against SGT model}

The Ro-dependence of the differences between HPE and SGT solutions was also used to validate the HPE model at larger timesteps. We increased the boundary-layer timestep from 10 to 15 minutes so that the variation of the boundary-layer diffusion between timesteps became large (at Ro $\simeq 0.1$ ). Methods of timestepping the boundary-layer physics in weather and climate 
models remains an active development area. Beljaars (1991) and

Cullen and Salmond (2003) proposed that a robust timestepping method should also preserve Ekman balance. Here, we compared three established methods of timestepping the boundary-layer diffusion:

1. Implicit. The increments due to the boundary layer were represented in the control HPE simulations by a standard implicit method, using implicit weights of one (Diamantakis et al. 2006). The weakness of the implicit method is that the change of diffusion across the timestep is not included.

2. K-update. The K-update method recalculates the diffusion at the end of the timestep and then repeats the implicit calculation (Cullen and Salmond 2003; Diamantakis et al. 2006). The updating of the diffusion means that all three terms in the Ekman balance are evaluated at the same time. At small Ro, the model is potentially closer to Ekman balance at the end of the time step (Beljaars 1991).

3. Wood et al. The re-calculation of diffusion is computationally expensive in operational models, so the method of Wood et al. (2007) approximates the non-linearity of the diffusion as

$$
K_{m} \propto \chi^{P+1}
$$

where $P$ is the non-linearity and $\chi$ represents any of $u, v$ or $\theta$. Here we used $P=1.5$, as recommended by Wood et al. (2007).

\section{Results}

\subsection{Baroclinic wave case}

In order to illustrate the control HPE simulations, here we describe their evolution for Ro $=0.15$. Figure 2 compares initial and 12-hour fields for the control HPE simulation. The initial wave was the fastest growing normal mode, with no boundary layer (Fig. 2a). The boundary-layer parametrization was part of the subsequent HPE integration. By 12 hours, the overall amplitude of the wave increased due to baroclinic instability and a boundary layer with a mean depth of $5 \%$ of the domain was established (Fig. 2b). Since this was a near-neutral scenario, the boundary-layer diffusion responded strongly to vertical shear of the horizontal (a) Initial $\widehat{v}$

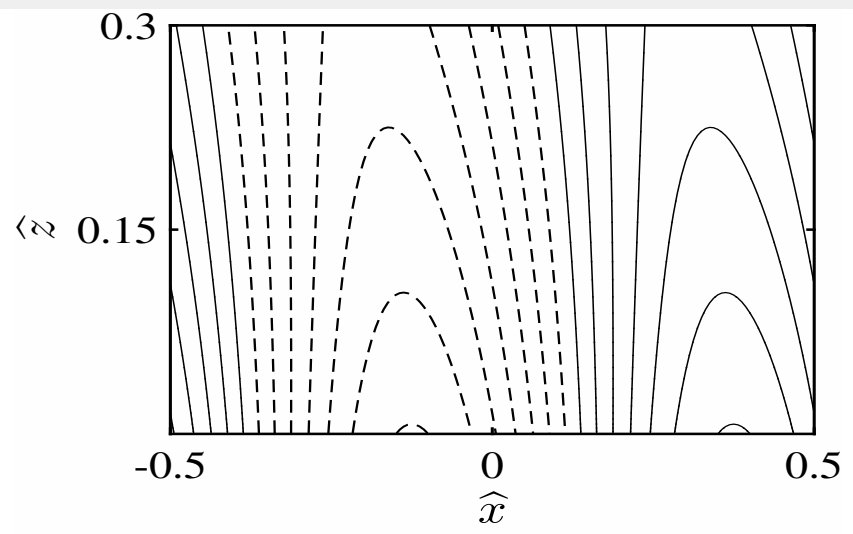

(b) $\widehat{v}$ at 12 hours

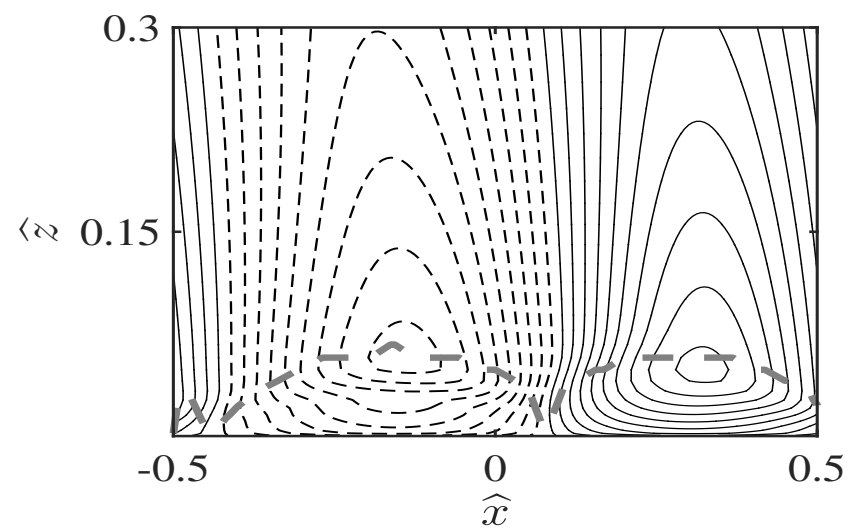

(c) $\widehat{\theta}$ at 12 hours

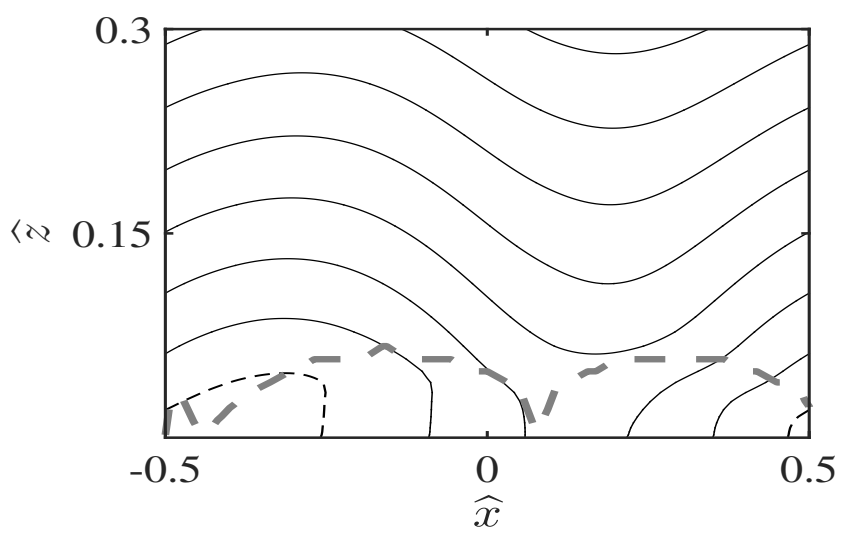

Figure 2. Vertical cross sections of control HPE simulation for Ro $=0.15$ : (a) Initial $v$-component of velocity, (b) $12 \mathrm{~h} v$-component of velocity and (c) $12 \mathrm{~h}$ potential temperature anomaly. Positive contours solid, negative values dashed. Boundary-layer top is grey-dashed line. All figures dimensionless and potential temperature normalised by $\frac{\partial \theta_{\text {ref }}}{\partial z} H=35 \mathrm{~K}$, where $\theta_{\text {ref }}$ is the background potential temperature. Contour interval 0.04 for wind and 0.05 for potential temperature.

wind. Thus, the maxima in boundary-layer depth corresponded closely to the extrema in the $v$-component of velocity.

Figure 2c shows the 12-hour potential temperature anomaly. Within the boundary layer, there were relatively low values on the left of the domain, but higher values on the right. The stratification varied across the domain, with shallow mixed layers to the left and stratified layers in the centre and to the right. Similar 
(a) Control HPE simulation, Ro $=0.15$.

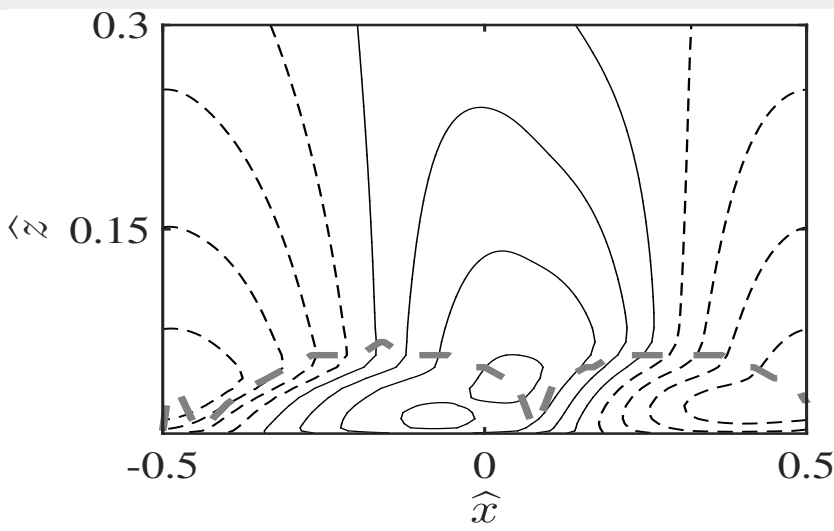

(b) SGT model, Ro = 0.15.

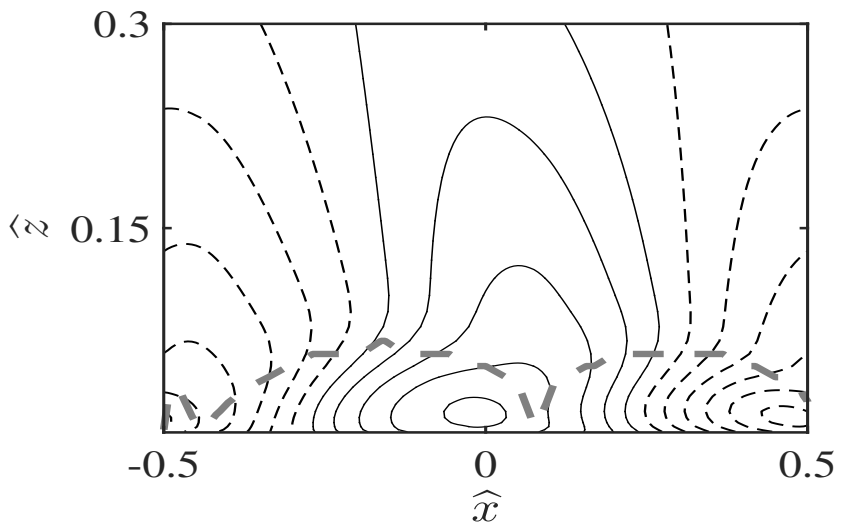

Figure 3. Vertical cross-sections of dimensionless ageostrophic wind for $\mathbf{R o}=\mathbf{0 . 1 5}$ (a) control HPE simulation and (b) diagnosed SGT solution. Contour interval 0.04 positive values solid, negative values dashed. Boundary-layer top grey-dashed line.

variations of stratification are found in more complex simulations of cyclogenesis (e.g. Beare 2007).

Figure 3a shows the ageostrophic wind for the control HPE simulation. Above the boundary layer there was a baroclinic wave. Within the boundary layer, positive maxima of ageostrophic wind corresponded to negative minima in the $v$-component of velocity (shown previously in Fig. 2b). The correlation was the familiar wind-turning effect. The control HPE simulation also exhibited substantial forward tilting of the ageostrophic wind. The tilting was due to the opposing effects of advection by the verticallysheared basic state horizontal wind and drag from the boundary layer. Similar tilting was reported by Keyser and Anthes (1982), although here the amplitude was smaller as the integrations were shorter.

\subsection{Differences between HPE and SGT models}

Above the boundary layer, the control HPE simulation (Fig. 3) was close to the SGT solution. However, differences remained within the boundary layer at Ro $=0.15$. Compared with the (a) HPE simulation, Ro $=0.037$.

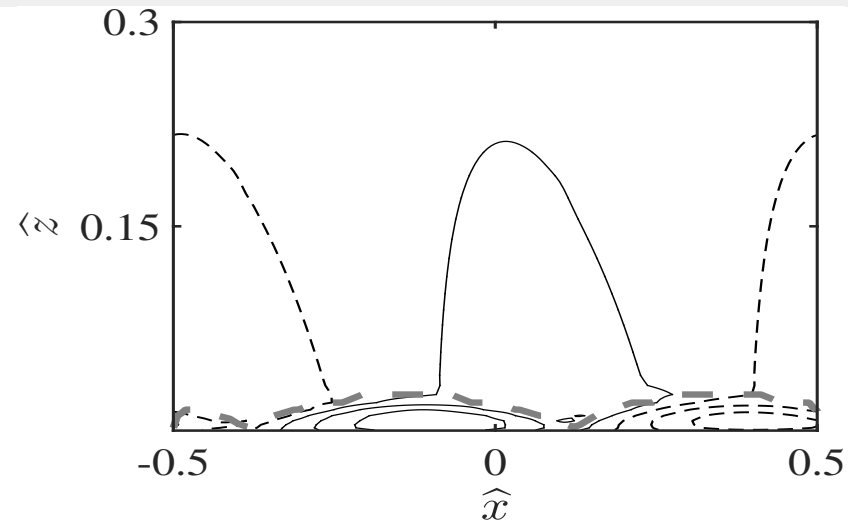

(b) SGT model, Ro = 0.037 .

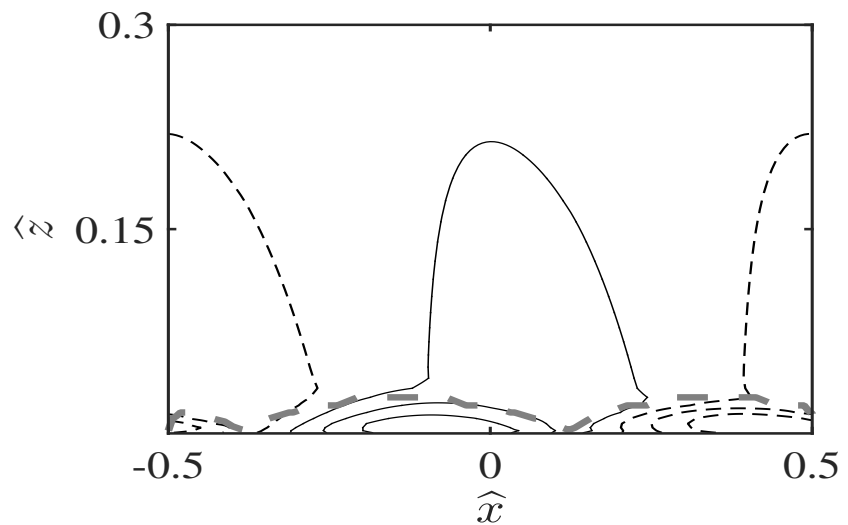

Figure 4. Vertical cross-section of dimensionless ageostrophic wind at $\mathbf{R o}=\mathbf{0 . 0 3 7}$ for the: (a) control HPE simulation and (b) diagnosed SGT solution. Contour interval 0.04, positive values solid, negative values dashed. Boundary-layer top indicated by grey-dashed line.

control HPE simulation, the SGT model was less tilted in the $x$ direction. Within the boundary layer, the ageostrophic wind of the SGT model was shifted to the right relative to the control HPE simulation. These differences were consistent with the reduced accuracy of the SGT assumption in the boundary layer compared with the free troposphere. It was also consistent with inaccuracies of the Ekman momentum approximation reported by Snyder (1998).

In contrast, at the smaller value of $\mathrm{Ro}=0.037$, the phase and orientation differences between the SGT model and control HPE simulation were markedly reduced (Fig. 4). The small differences provide clear evidence of convergence of the control HPE simulation to the SGT solution with decreasing Ro. Also, the boundary-layer depth decreased substantially, indicating its dependence on Ro, as proposed in Section 2.

We now combine the results of 13 control HPE simulations with Ro in the range of 0.037 to 0.15 . Figure 5 shows encouraging convergence of the control HPE simulations to the SGT solution 


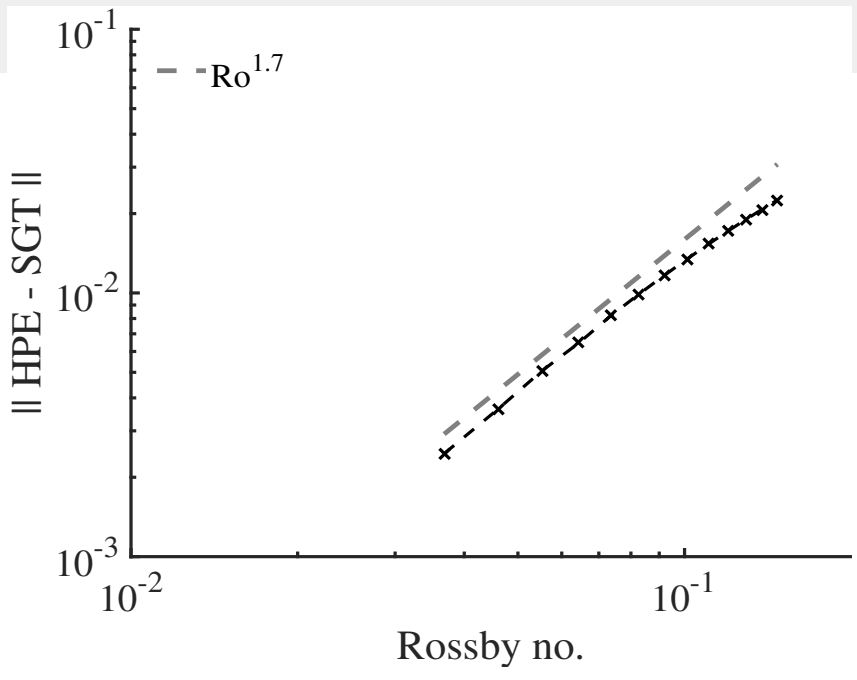

Figure 5. Rms difference between HPE and SGT solutions averaged over entire model domain and plotted against Ro (log-log plot). The $\mathrm{Ro}^{1.7}$ line is grey-dashed.

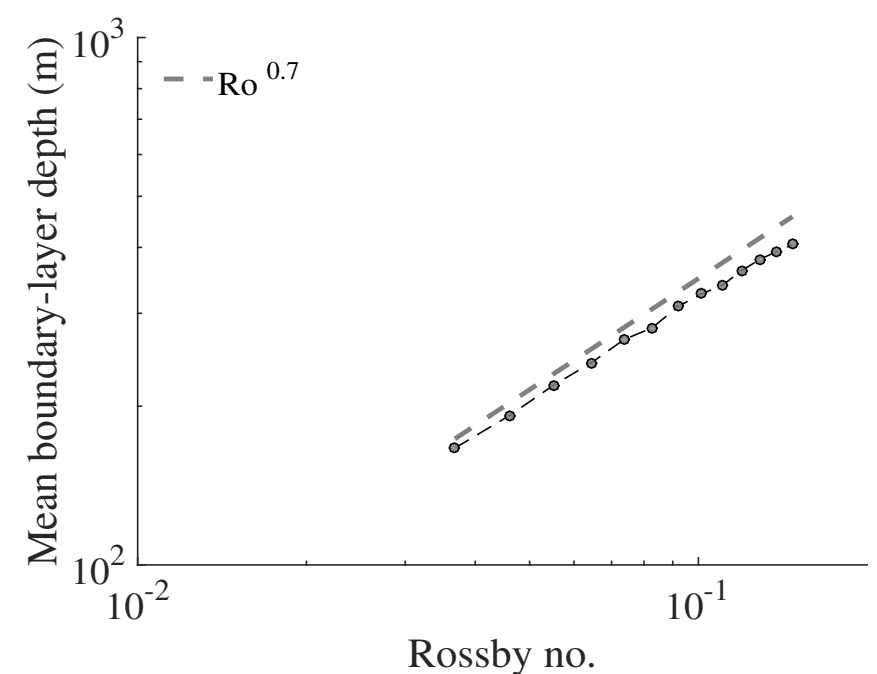

Figure 6. Domain-averaged boundary-layer depth $(\bar{h})$ against Ro (log-log plot). The Ro ${ }^{0.7}$ line is grey-dashed.

with decreasing Ro. Below Ro $=0.1$, the norm of the difference tended to $\mathrm{Ro}^{1.7}$.

\subsection{Understanding the convergence of HPE solutions to SGT}

We now interpret the convergence rate in Fig. 5. The variation of mean boundary-layer depth with Ro is shown in Fig. 6. There was a clear power law for Ro $<0.1$ and the exponent was $m=0.7$ (Eq. 28). In agreement with our scale assumptions, the difference between the HPE and SGT solutions varied as the product of the mean boundary-layer depth and Ro (Eq. 27). The convergence rate was thus determined by the boundary layer.

It was instructive to separate the domain-averaged differences between the HPE and SGT solutions into averages within the boundary layer and above it (Fig. 7). The convergence of the HPE to the SGT solutions was first-order within the boundary layer, but second-order above it. Within the boundary layer, the

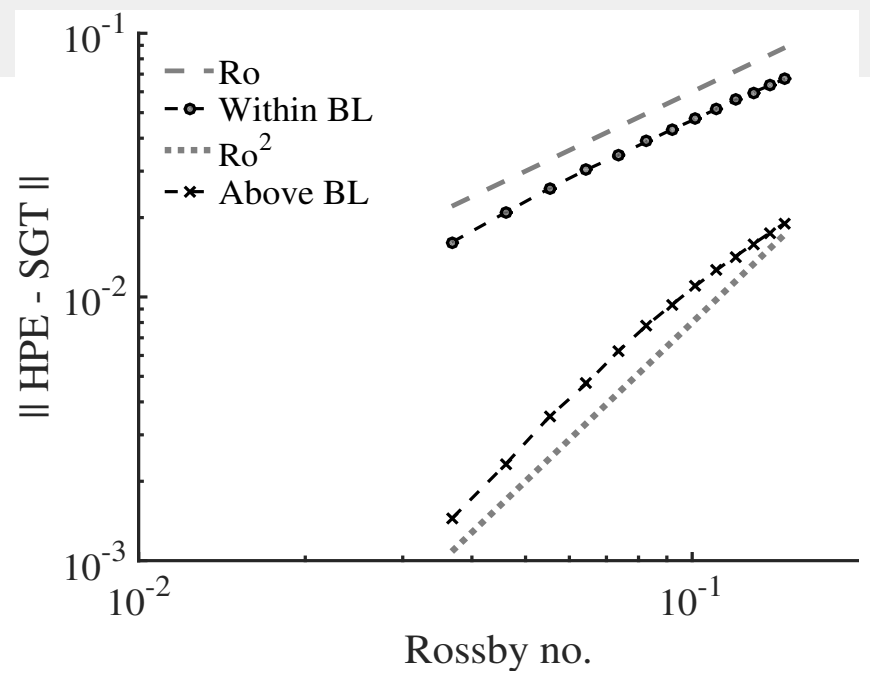

Figure 7. Differences between HPE and SGT solutions split into averages within and above the boundary layer. The Ro line grey-dashed. The $\mathrm{Ro}^{2}$ line grey-dotted.

first-order assumptions controlled the convergence rate; here, the boundary-layer depth was implicit in the averaging calculation, so its dependence on Ro was factored out. Above the boundary layer, the second-order approximation of the material derivative dominated below Ro $=0.1$. The second-order convergence rate was in agreement with that found for the dynamics-only SG case during the early phases of baroclinic development (Cullen 2008). These findings were in agreement with the estimates made in Eqs. (21) and (23).

The comparisons between the SGT model and HPE simulations in Figs. 3 and 4 can be understood further from the momentum budgets. Figure 8 compares momentum budgets for the control HPE simulations within the boundary layer. For Ro $=0.15$ (Fig. 8a), advection was important, and the Ekman balance between the Coriolis and drag terms was not a good approximation. Given that the SGT model relaxed back to Ekman balance in the boundary layer, it could not be expected to be a good approximation to the HPE simulations at Ro $=0.15$ (Fig. 3). However, for Ro $=0.037$, the advection became a small component and Ekman balance was valid (Fig. 8b). The agreement between the HPE simulations and the SGT model was now much better in the boundary layer.

\subsection{Validating large-timestep HPE simulations against the}

\section{SGT model}

We repeated the HPE simulations at a larger boundary-layer timestep of 15 mins (previously 10 mins). The variation of the boundary-layer diffusion between timesteps became important at Ro of about 0.1. Such a regime is typical in operational 
(a) $\operatorname{Ro}=0.15$

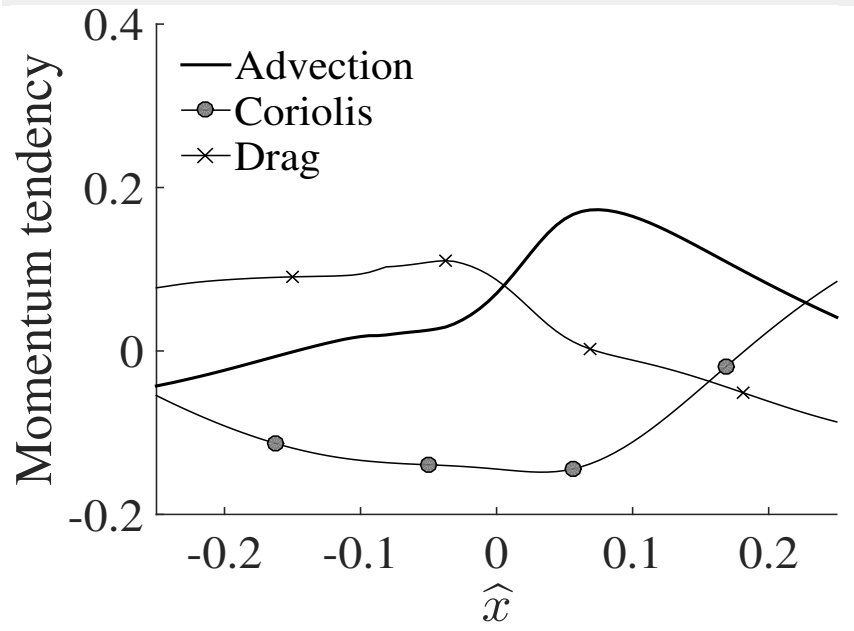

(b) $\operatorname{Ro}=0.037$

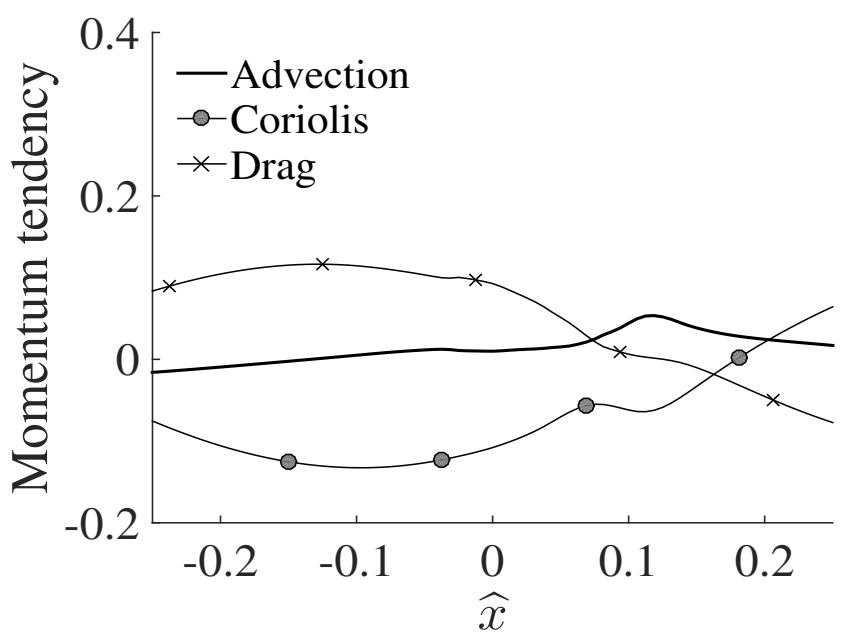

Figure 8. Momentum budgets in $y$-direction for control HPE simulations and middle of the boundary layer $(z=\bar{h} / 2)$ for two values of Ro. Momentum tendencies non-dimensionalised by $f u_{0}$ and plotted against $\widehat{x}$. Coriolis refers to the $-f\left(u-u_{q}\right)$ term

weather and climate models. Figure 9 shows cross-sections of the ageostrophic winds from the HPE model for the Implicit and Kupdate schemes. Whilst the K-update fields were not perfectly smooth, they improved markedly on the Implicit scheme. The Implicit scheme gave substantial small-scale noise within the boundary layer; the small scales were inconsistent with those required for Ekman balance, as described in section 2.

The SGT fields diagnosed from the control HPE simulations were now used to validate the HPE simulations at larger timesteps. If the larger-timestep HPE simulations and the SGT model did not converge at the rate previously calculated, it indicated numerical problems. Figure 10 shows the difference between the HPE simulations using different timestepping schemes and the SGT model. At smaller values of Ro, all HPE models followed the ideal Ro ${ }^{1.7}$ line. However, above Ro $=0.08$, the HPE model (a) Implicit

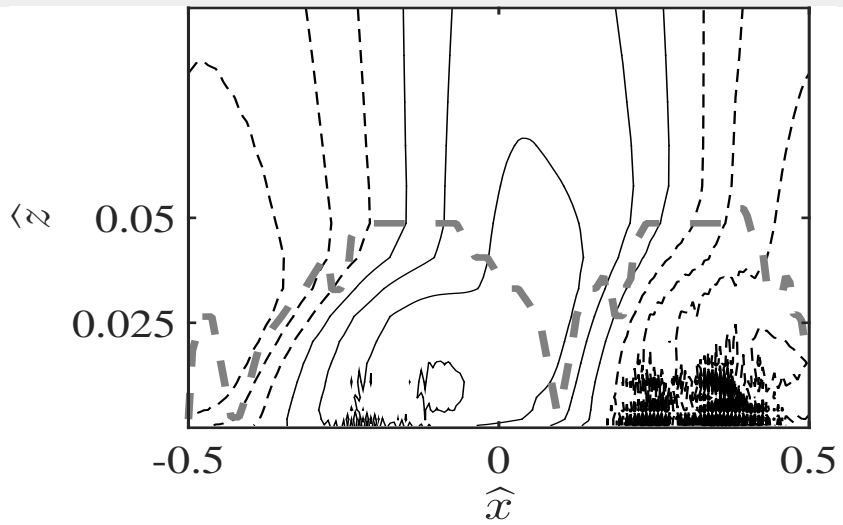

(b) K-update

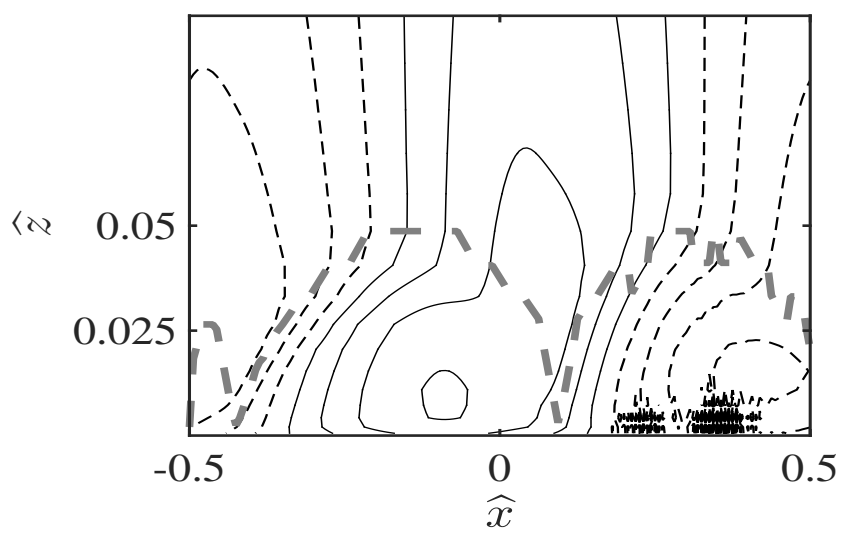

Figure 9. Vertical cross-section of ageostrophic winds from the HPE model with boundary-layer timestep 15 mins and Ro $=0.1$. (a) Implicit and (b) K-update schemes. Contour interval 0.04 , positive values solid, negative values dashed. Boundary-layer top grey-dashed line.

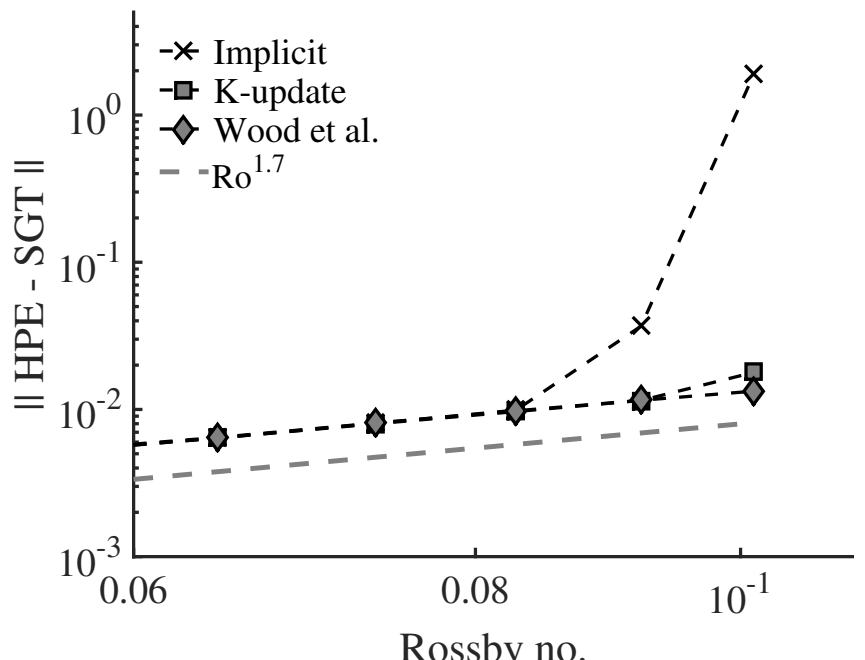

Figure 10. The difference between HPE model with boundary-layer timestep 15 mins and SGT model (rms domain average) against Ro (log-log plot). Shown for 3 methods of boundary-layer timestepping: Implicit, K-update and Wood et al. (2007). The Ro ${ }^{1.7}$ line is grey-dashed.

using the Implicit scheme started to deviate markedly above the ideal line, and no longer converged at the required rate. The HPE model using the K-update scheme deviated slightly above the ideal line at Ro $=0.1$. The HPE model using the Wood et al. (2007) scheme followed the ideal $\mathrm{Ro}^{1.7}$ line for the range of Ro shown. Both the K-update and Wood et al. (2007) schemes accounted for 
the variation of the boundary-layer diffusion across the timestep, giving improved convergence properties compared to the Implicit scheme.

\section{Discussion}

In this paper, we presented a new way of validating baroclinicwave simulations coupled to a boundary-layer parametrization. Our study represents one example of the more general problem of physics-dynamics coupling: understanding how physical parametrizations couple to the resolved dynamics (Staniforth et al. 2002; Cullen and Salmond 2003). Physics-dynamics coupling presents many challenges for the future development of weather and climate models. Our new test involved running HPE models at the small-Ro limit, and comparing with a balanced model that included a boundary layer: the SGT model. Previous work focused on the convergence of the HPE solutions to SG solutions for the dynamics-only case (Cullen 2008; Visram et al. 2014). For the first time, we determined the role of the boundary-layer parametrization in modifying the dynamics-only results. Our key findings were:

1. When the timescale was controlled by advection, Ro controlled the convergence of the HPE solutions to SGT.

2. The domain-averaged rms difference between HPE and SGT solutions varied as the product of Ro and the mean boundary-layer depth; for our cases, the difference varied as $\operatorname{Ro}^{1.7}$.

3. The $\operatorname{Ro}^{1.7}$ dependence was also used to validate the HPE model at a larger timestep. The convergence rate was disrupted for a standard implicit timestepping scheme, but improved for schemes that accounted for the change of the boundary-layer diffusion across the timestep.

The comparison of the HPE and SGT models over a range of Ro has not been done before. The SGT model extended the SG model by including a realistic boundary layer close to Ekman balance. In addition to averaging over the entire domain, we found it useful to consider separate averages over the boundary layer and free troposphere. The SGT model was first-order accurate within the boundary layer, because it was strongly constrained by Ekman balance. However, it was second-order accurate in the free troposphere, in agreement with the dynamics-only case (Cullen 2008; Visram et al. 2014). Thus the convergence of HPE solutions to SGT model was dominated by the differences in the boundary layer. However, the boundary-layer depth was shown to decrease with Ro, improving the domain averaged convergence rate.

Perhaps the closest study to ours is Snyder (1998). There, he diagnosed the Ekman momentum approximation from a $2 \mathrm{D}$ HPE frontal simulation including a boundary layer. He concluded that the Ekman momentum approximation was poor in frontal scenarios. In contrast, here we considered the early phases of baroclinic development, in the limit of small Ro. The benefit of the SGT model was found to be greater in these situations. The reduced accuracy of the SGT model within the boundary layer provided a caution against its use at values of Ro above about 0.1 . For example, the accuracy of the SGT model would be poor at sharp fronts, in agreement with Snyder (1998).

We have avoided the need to compute solutions of the SGT model prognostically in this study by using a diagnostic technique. This is used to show whether HPE solutions scale with Ro in the manner expected by theory. As discussed in Cullen (2007), if a solution of a reduced model like SGT exists, it is possible to prove that solutions of the full equations converge to it, possibly with prepared initial data. While the necessary mathematical theory has not yet extended to this case, we have demonstrated the expected rates of convergence. This adds support to the theoretical estimates as well as suggesting that the control HPE solutions have the correct large-scale behaviour. The large timestep tests show that this behaviour can be degraded by inaccurate numerical techniques.

The approach here could also be applied to testing either alternative parametrizations or different dynamical regimes other than a baroclinic wave. For example, Ekman balance still applies in the boundary layer even when the Coriolis parameter is zero (Beare and Cullen 2012). Thus equatorial scenarios could be investigated. Many challenges for weather and climate models involve their physics-dynamics couplings. The validation approach used here could be employed with more complicated numerical weather prediction models. For example, the role of the grid, advection scheme and numerical coupling of the advection and boundary-layer schemes could be investigated. Using an 
idealised baroclinic wave case for the Met Office Unified Model

(Beare 2007), the geostrophic winds and Coriolis parameter could be rescaled simply as shown here. Thus, the convergence to the SGT model could be used to validate a full numerical weather prediction model.

\section{Acknowledgement}

We thank Professor Nigel Wood of the Met Office for his feedback on the paper.

\section{Appendix}

\subsection{Boundary-layer scheme}

The boundary-layer scheme used vertical diffusions of momentum and heat expressed as a function of a mixing length $(\lambda)$, the vertical wind shear $(S)$ and functions of gradient Richardson number $(\mathrm{Ri})$

$$
\begin{array}{r}
K_{m}=\lambda^{2} S f_{m}(\mathrm{Ri}), \quad K_{h}=\lambda^{2} S f_{h}(\mathrm{Ri}), \\
S^{2}=\left(\frac{\partial u}{\partial z}\right)^{2}+\left(\frac{\partial v}{\partial z}\right)^{2}, \quad R i=\frac{g}{S^{2} \theta_{0}} \frac{\partial \theta}{\partial z},
\end{array}
$$

where the mixing length was defined as

$$
\frac{1}{\lambda}=\frac{1}{\lambda_{0}}+\frac{1}{\kappa\left(z+z_{0}\right)}
$$

and $\lambda_{0}=40 \mathrm{~m}, \kappa=0.4$ is the von-Karman constant and $z_{0}=0.1 \mathrm{~m}$ the roughness length. The terms $f_{m}$ and $f_{h}$ are stability functions for momentum and heat respectively and defined separately for stable and unstable stratification

$$
f_{m}(R i)=\operatorname{Pr} f_{h}(R i)= \begin{cases}1 /(1+10 R i) & \mathrm{Ri} \geq 0 \\ (1-16 R i)^{1 / 2} & \mathrm{Ri}<0\end{cases}
$$

where $\operatorname{Pr}=0.7$ is the neutral Prandtl number. A two-time-level scheme was used for the boundary layer parametrization. In the control set-up, an implicit timestepping method was used for the boundary layer.

We now summarise the methods of diagnosing velocities for the: Ekman balance, SGT model and initial ageostrophic circulations

\subsection{Calculating Ekman-balanced velocity}

Since the boundary-layer drag term was evaluated implicitly, an alternative evaluation of $\left(u_{e}, v_{e}\right)$ to that used by Beare and Cullen (2013) was required. Instead, we made a first evaluation of $\left(u_{e}, v_{e}\right)$, by setting the material derivative to zero in Eqs. (32) and (34) and keeping the boundary-layer drag fixed. We then recalculated the boundary-layer terms, and iterated further if required. 


\subsection{Sawyer-Eliassen equation}

The standard SG Sawyer-Eliassen equation was used to initialise the ageostrophic circulation in the model

$f\left(f+\frac{\partial v_{g}}{\partial x}\right) \frac{\partial^{2} \psi^{\prime}}{\partial z^{2}}-2 \frac{g}{\theta_{0}} \frac{\partial \theta}{\partial x} \frac{\partial^{2} \psi^{\prime}}{\partial x \partial z}+N^{2} \frac{\partial^{2} \psi^{\prime}}{\partial x^{2}}=2 \frac{\partial v_{g}}{\partial x} \frac{g}{\theta_{0}} \frac{\partial \Theta}{\partial y}$,

where

$$
\left(u_{a}, w\right)=\left(\frac{\partial \psi^{\prime}}{\partial z},-\frac{\partial \psi^{\prime}}{\partial x}\right)
$$

The boundary conditions on vertical velocity gave

$$
\psi^{\prime}=0 \quad \text { at } z=0 \text { and } z=H,
$$

where $\psi^{\prime}$ was periodic in $x$.

\subsection{Calculating SGT model velocity}

Beare and Cullen (2013) derived a Sawyer-Eliassen circulation equation including a boundary layer using the SGT model. The equation was derived by requiring that Ekman balance was maintained in the time evolution. We also included baroclinic forcing here to give

$$
\mathcal{L} \psi=\overbrace{\left(f^{2}+\mathcal{D}^{2}+\frac{\partial \mathcal{D}}{\partial t}\right) \frac{\partial u_{e}}{\partial z}-\frac{\partial \mathcal{F}_{b}}{\partial x}}^{\text {Boundary layer }}+\overbrace{\left(\frac{\partial v_{e}}{\partial x}+\frac{\partial v}{\partial x}\right) \frac{g}{\theta_{0}} \frac{\partial \Theta}{\partial y}}^{\text {Baroclinic }}
$$

where the operator, $\mathcal{D}$, applied to an an arbitrary function, $g$, is written as $\mathcal{D} g=\frac{\partial^{2}}{\partial z^{2}}\left(K_{m} g\right)$ and

$$
\mathcal{F}_{b}=\frac{g}{\theta_{0}} \frac{\partial}{\partial z}\left(K_{h} \frac{\partial \theta}{\partial z}\right)
$$

The operator $\mathcal{L}$ in Eq. (51) is given by

$$
\begin{array}{r}
\mathcal{L} \psi=\left[f\left(f+\frac{\partial v_{e}}{\partial x}\right)+\mathcal{D}^{2}+\frac{\partial u_{e}}{\partial x} \mathcal{D}\right] \frac{\partial^{2} \psi}{\partial z^{2}}+N^{2} \frac{\partial^{2} \psi}{\partial x^{2}} \\
-\left(\frac{\partial u_{e}}{\partial z} \mathcal{D}-\mathcal{D} \frac{\partial u_{e}}{\partial z}+2 \frac{g}{\theta_{0}} \frac{\partial \theta}{\partial x}\right) \frac{\partial^{2} \psi}{\partial x \partial z}-\frac{\partial \mathcal{D}}{\partial x} \frac{\partial u_{e}}{\partial z} \frac{\partial \psi}{\partial z} \\
+\frac{\partial \mathcal{D}}{\partial z} \frac{\partial u_{e}}{\partial z} \frac{\partial \psi}{\partial x} .
\end{array}
$$

The streamfunction $\psi$ was defined as

$$
\left(u_{s}-u_{g}, w_{s}\right)=\left(\frac{\partial \psi}{\partial z},-\frac{\partial \psi}{\partial x}\right)
$$

The vertical velocity was zero on the top and bottom boundaries, giving

$$
\psi=0 \quad \text { at } z=0 \text { and } z=H,
$$

and $\psi$ was periodic in $x$. The method for calculating $v_{s}$ was as Beare and Cullen (2013).

\section{References}

Bannon PR. 1998. A comparison of Ekman pumping in approximate models of the accelerating Planetary Boundary Layer. J. Atmos. Sci. 55: 1446-1451.

Batchelor G. 2000. An introduction to fluid dynamics. Cambridge Mathematical Library, Cambridge University Press, ISBN 9780521663960. Beare RJ. 2007. Boundary layer mechanisms in extratropical cyclones. Quart. J. Roy. Meteorol. Soc. 133: 503-515.

Beare RJ, Cullen MJP. 2010. A semi-geostrophic model incorporating wellmixed boundary layers. Quart. J. Roy. Meteorol. Soc. 136: 906-917.

Beare RJ, Cullen MJP. 2012. Balanced models of boundary-layer convergence. Quart. J. Roy. Meteorol. Soc. 138: 1452-1464.

Beare RJ, Cullen MJP. 2013. Diagnosis of boundary-layer circulations. Phil Trans $R$ Soc A 371: 20110474: 1-14.

Beare RJ, MacVean MK, Holtslag AM, Cuxart J, Esau I, Golaz JC, Jimenez MA, Khairoutdinov M, Kosovic B, Lewellen D, Lund TS, Lundquist JK, McCabe A, Moene AF, Noh Y, Raasch S, Sullivan P. 2006. An intercomparison of large-eddy simulations of the stable boundary layer. Boundary-Layer Meteorol. 118: 247-272.

Beljaars ACM. 1991. Numerical schemes for parametrizations. Proceedings of the ECMWF seminar on numerical methods in atmospheric models : 308334.

Cullen MJP. 1989. On the incorporation of atmospheric boundary layer effects into a balanced model. Quart. J. Roy. Meteorol. Soc. 115: 1109-1131.

Cullen MJP. 2006. A mathematical theory of large-scale atmosphere/ocean flow. Imperial College Press, 259 pp. .

Cullen MJP. 2007. Modelling atmospheric flows. Acta Numerica 16: 67-154. Cullen MJP. 2008. A comparison of numerical solutions to the Eady frontogenesis problem. Quart. J. Roy. Meteorol. Soc. 134: 2143-2155.

Cullen MJP, Salmond DJ. 2003. On the use of a predictorcorrector scheme to couple the dynamics with the physical parametrizations in the ecmwf model. Quart. J. Roy. Meteorol. Soc. 129: 12171236.

Diamantakis M, Wood N, Davies T. 2006. An improved implicit predictorcorrector scheme for boundary layer vertical diffusion. Quart. J. Roy. 
Meteorol. Soc. 132: 959-978.

Eliassen A. 1962. On the vertical circulation in frontal zones. Geophys. Publ.

24: $147-160$.

Garratt JR. 1992. The atmospheric boundary layer. Cambridge University Press, 316pp. .

Hoskins BJ, Bretherton FP. 1972. Atmospheric frontogenesis models: mathematical formulation and solution. J. Atmos. Sci. 29: 11-37.

Keyser D, Anthes RA. 1982. The influence of planetary boundary layer physics on frontal structure in the Hoskins-Bretherton horizontal shear model. J. Atmos. Sci. 39: 1783-1802.

Ostdiek V, Blumen W. 1997. A dynamic trio: Inertial oscillation, deformation frontogenesis. J. Atmos. Sci. 54: 14901502.

Sawyer JS. 1956. The vertical circulation at meteorological fronts and its relation to frontogenesis. Proc. R. Soc. London. A234: 346-362.

Snyder C. 1998. Approximate dynamical equations for fronts modified by the planetary boundary layer. J. Atmos. Sci. 35: 777-787.

Staniforth A, Wood N, Cote J. 2002. Analysis of the numerics of physicsdynamics coupling. Quart. J. Roy. Meteorol. Soc. 128: 2779-2799.

Tory KJ, Reeder MJ. 2005. The effect of the continental boundary layer on the dynamics of fronts in a 2D model of baroclinic instability. I: An insulated lower surface. Quart. J. Roy. Meteorol. Soc. 131: 2389-2408.

Visram A, Cotter CJ, Cullen MJP. 2014. A framework for evaluating model error using asymptotic convergence of the Eady model. Quart. J. Roy. Meteorol. Soc. 140: 1629-1639.

Williams RT. 1967. Atmospheric frontogenesis: a numerical experiment. $J$. Atmos. Sci. 24: 627-641.

Wood N, Diamantakis M, Staniforth A. 2007. A monotonically-damping second-order-accurate unconditionally-stable numerical scheme for diffusion. Quart. J. Roy. Meteorol. Soc. 133: 1559-1573. 